\title{
Effect of Coronal Mass Ejection on Earth's Magnetic Field during Ascending Phase of Solar Cycles 23-24
}

\section{Bharti Nigam1, Prithvi Raj Singh², Pramod Kumar Chamadia1, Ajay Kumar Saxena², Chandra Mani Tiwari²}

${ }^{1}$ Govt. Autonamous P. G. College, Satna, India

${ }^{2}$ Department of Physics, Awadhesh Pratap Singh University, Rewa, India

Email: Id- bharti.nigam@gmail.com, prithvisingh77@gmail.com

How to cite this paper: Nigam, B., Singh, P.R., Chamadia, P.K., Saxena, A.K. and Tiwari, C.M. (2017) Effect of Coronal Mass Ejection on Earth's Magnetic Field during Solar Cycles 23-24. International Journal ot Astronomy and Astrophysics, 7, 213-220. https://doi.org/10.4236/ijaa.2017.73017

Received: August 31, 2017

Accepted: September 15, 2017

Published: September 18, 2017

Copyright ( $) 2017$ by authors and Scientific Research Publishing Inc. This work is licensed under the Creative Commons Attribution International License (CC BY 4.0).

http://creativecommons.org/licenses/by/4.0/

\section{c) (i) Open Access}

\begin{abstract}
We have studied the width and speed of coronal mass ejections (CMEs) and geomagnetic disturbance storm time (Dst) Index during ascending phase of solar cycles 23 and 24 . We have classified total CMEs according to angular width and speed for the ascending period 1996-2002 and 2008-2014. We have found that the width of $62 \%$ CMEs is narrow, and $3 \%$ are Halo for the solar cycle 23 and 73\% CMEs are narrow, and 2\% CMEs are Halo for the solar cycle 24. The speed distribution of $65 \%$ CMEs has speed $\leq 500 \mathrm{~km} / \mathrm{sec}$ and $4 \%$ CMEs has speed $>1000 \mathrm{~km} / \mathrm{sec}$ for solar cycle 23 and $84 \%$ CMEs has speed $\leq$ $500 \mathrm{~km} / \mathrm{sec}$ and $1 \%$ CMEs has speed $>1000 \mathrm{~km} / \mathrm{sec}$ in cycle 24 . The relationship between width and speed is more pronounced for fast ejecta $(>1000$ $\mathrm{km} / \mathrm{sec}$.), while slower ejecta shows more astronomically immense scatter. We have reported that the correlation between Dst and CMEs for ascending phase of solar cycle 24 is less than as compare to ascending phase of solar cycle 23.
\end{abstract}

\section{Keywords \\ CME, Dst, SSN}

\section{Introduction}

Now it is very well known that the coronal mass ejections (CMEs) play a central role in the enhancing of interplanetary and geomagnetic activity [1] [2] [3]. The disturbance storm time index (Dst) is a measure of geomagnetic activity used to assess the magnetic storms and it is affected by solar output. Many researchers show the relationship between CMEs and geomagnetic storms [4] [5] [6] [7]. Coronal mass ejections (CMEs) are identified in the images of the solar corona 
obtained by the Solar and Heliospheric Observatory (SOHO) mission's Large Angle and Spectrometric Coronagraph (LASCO) since 1996. Coronal mass ejections (CMEs) with strong magnetic fields caused geomagnetic storms [8].

CMEs travel outward from the Sun, typically at an average speed of about $\sim 483$ kilometers per second but be slow as $\sim 100$ kilometers per second or faster than $\sim 3000$ kilometers per second [9] [10]. The fastest CMEs erupt from large sunspot active regions, powered by the strongest magnetic field concentrations on the Sun. These fast CMEs can reach Earth in a little time as 14 - 17 hours and caused major geomagnetic storms. It is well-known that the most solar cycles show a double peak due to the out-of-phase activity in the two hemispheres [11]. The double peak in SSN during solar cycle 24 is the second peak is larger than the first one by $\sim 20 \%$. Such a behavior was observed only a few times since the 1800s [12].

In order to address the fundamental problem of prediction of geomagnetic storms, it is important to examine the Halo CMEs first observed by Howard [13]. A Halo CMEs appears to surround the occulting disk of the observing coronagraph in sky plane projection. Halo CMEs constitute only approximate $3 \%$ of all CMEs and represent an energy population because most of the CMEs that produce major geomagnetic storms are Halo [14]. There only 11 CMEs that caused major storms during cycle 24 until the end of period 2014 [15]. CMEs number shows a double peak and similar to seen in sunspot number [16].

In this study, we analyze the correlation between Dst and CMEs (Fast and Halo) during the ascending phase of solar cycles 23 and 24. In this paper, we have studied the variation of Dst and CMEs (speed and width) for the selected period.

\section{Data Detection and Method of Analysis}

We have taken CMEs and Dst data during the ascending phase of solar cycle 23 and 24. We have selected the first 7 years of both solar cycles 23 and 24 i.e. (1996-2002 and 2008-2014). We found total 7180 CMEs events in solar cycle 23 and 11,690 CMEs in solar cycle 24 for our selected period. In the present study, we have selected CMEs events data on the basis of angular width and speed from SOHO/LASCO (cdaw.gsfc.nasa.gov/CME_list/). In this analysis, we have distributed CMEs events in Narrow (width $\leq 60^{\circ}$ ), Moderate (width $61^{\circ} \sim 120^{\circ}$ ), Partial Halo (width $121^{\circ} \sim 359^{\circ}$ ), and Halo (width $=360^{\circ}$ ). For speed distribution we have classified CMEs in such categories as $\leq 500 \mathrm{~km} / \mathrm{sec}, 501 \sim 1000 \mathrm{~km} / \mathrm{sec}$, $1000 \sim 1500 \mathrm{~km} / \mathrm{sec},>1500 \mathrm{~km} / \mathrm{sec}$. The values of the annual average of geomagnetic disturbance storms time Index (Dst) (based on daily data) from the OmniWeb (https://omniweb.gsfc.nasa.gov/form/dx1.html).

\section{Results and Discussion}

According to speed distribution, we have taken the ascending phase of solar cycle 23 and 24.We have considered the interval 1996 to 2002 for solar cycle 23 and 2008 to 2014 for solar cycle 24 . We have divided CMEs events in speed wise into four categories 1) CMEs with speed $\leq 500 \mathrm{~km} / \mathrm{sec}$ 2) CME with speed $501 \sim$ 
$1000 \mathrm{~km} / \mathrm{sec} 3$ ) CMEs with speed $1001 \sim 1500 \mathrm{~km} / \mathrm{sec}$ 4) CMEs with speed > $1500 \mathrm{~km} / \mathrm{sec}$. Figure 1 shows the pie diagram for the distribution of CMEs according to above four categories for solar cycles 23 and 24. From Figure 1 we have observed that the CMEs with speed $\leq 500 \mathrm{~km} / \mathrm{sec}$ for solar cycle 23 is $65 \%$ and for solar cycle 24 is $84 \%$. It is noted that the CMEs with speed $\leq 500$ are larger in solar cycle 24 as compared to solar cycle 23.

Figure 2(a) shows the annual occurrence of fast CMEs (>1000) during the solar cycles 23 and 24. From Figure 2(a) it is found that the fast CMEs have double peaks in the ascending phase of solar cycle 23 (2000 \& 2002) and the ascending phase of solar cycle 24 (2012 \& 2014). We have observed that the time-lag for fast CMEs $(>1000)$ between the first maximum to the second
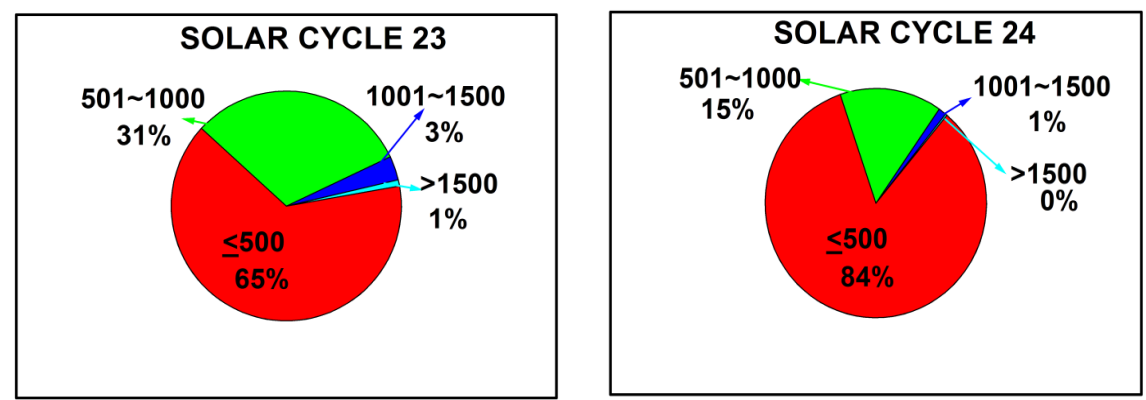

Figure 1. Shows the distribution of total CMEs according to CME's speed for ascending phase of solar cycles 23 and 24 .

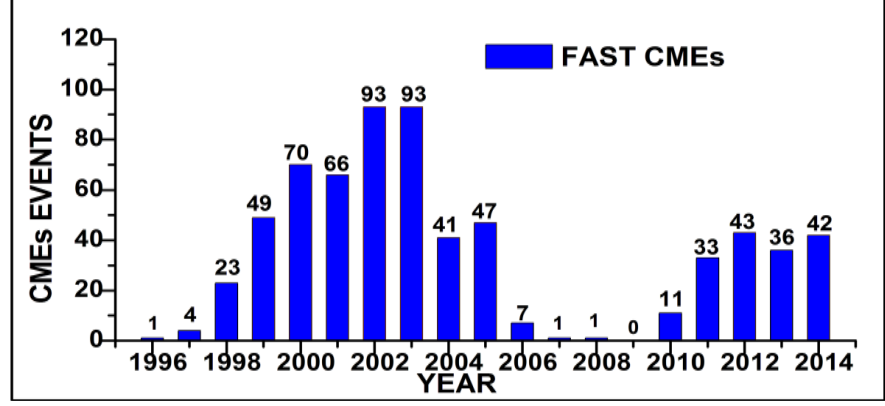

(a)
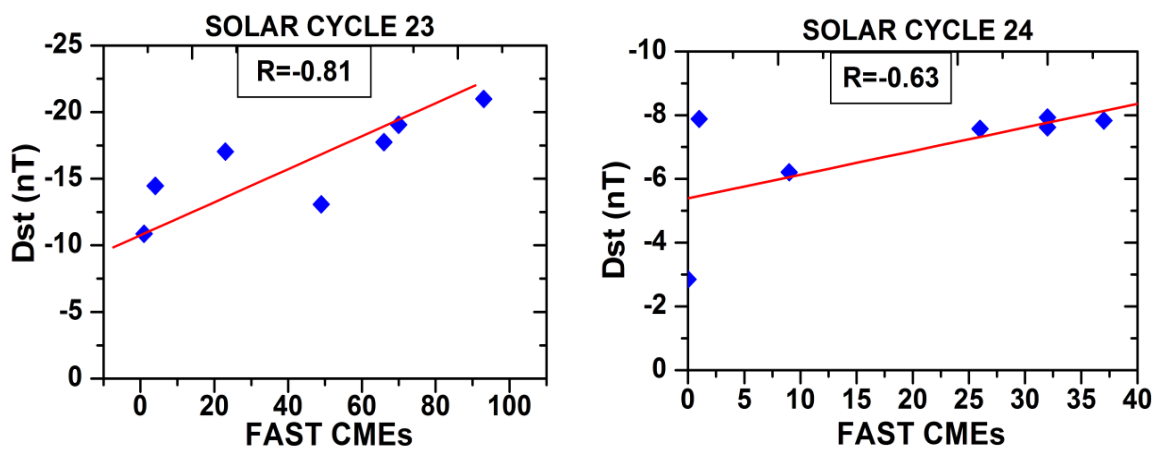

(b)

Figure 2. (a) Shows the graph of annual occurrence of fast CMEs during solar cycles 23 and 24 (b) scatter graph between fast CMEs and Dst Index during ascending phase of solar cycle 23 and 24 . 


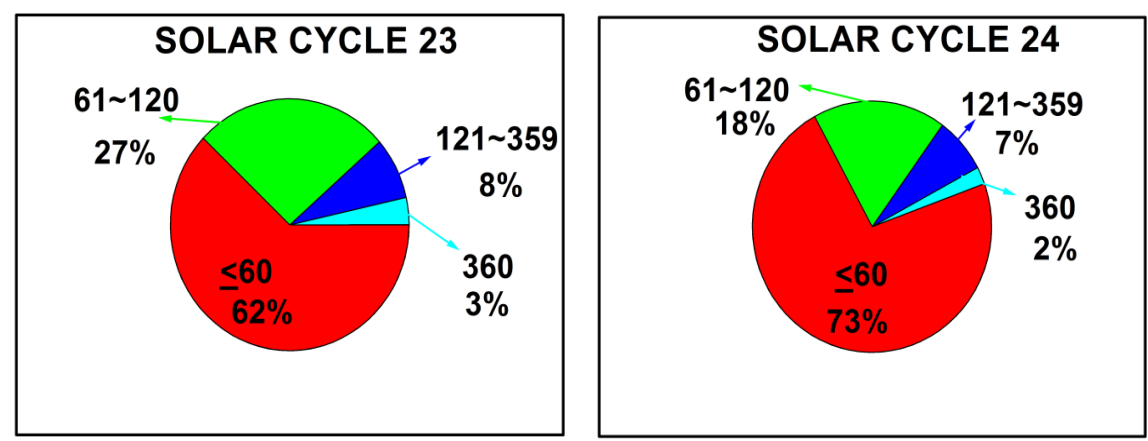

Figure 3. Shows the distribution of total CMEs according to Angular width for the ascending phase of solar cycles 23 and 24 .

maximum is $\sim 1$ years during the ascending phase of solar cycles 23 and 24 . Fast CMEs are often causing major Geomagnetic storms (Figure 2(b)). Figure 2(b) shows the scatter graph between fast CMEs and Dst index. The correlation coefficient between fast CMEs and Dst index for solar cycle 23 is $R=-0.81$ and for solar cycle 24 is $R=-0.63$. From Figure 2 (b) we have found that the correlation coefficient between Dst and fast CMEs (>1000) is larger in ascending phase of solar cycle 23 as compared to cycle 24 . We have reported that the solar activity is larger in cycle 23 as compared to cycle 24 .

From Figure 3 we have divided all annual occurrence of total CME events for angular width in following four categories for the ascending period of 1996-2002 and 2008-2014: (1) Narrow (width $\leq 60^{\circ}$ ) (2) Moderate (width $61^{\circ} \sim 120^{\circ}$ ) (3) Partial Halo (width $121^{\circ} \sim 359^{\circ}$ ) (4) Halo (width $=360^{\circ}$ ). From Figure 3 we have observed that the Narrow CMEs have the highest percentage in total CMEs during the ascending phase of solar cycles 23 and 24. In total, Halo CMEs are only $3 \%$ in solar cycle 23 and only $2 \%$ in solar cycle 24 .

Annual occurrence of Halo CMEs is shown in Figure 4(a) during solar cycles 23 and 24. It is clear that Annual occurrence of Halo CMEs has a double peak structure in solar cycles 24 (2012 \& 2014). From Figure 4(a) we have found that the time-lag for Halo CMEs (width $=360^{\circ}$ ) between the first maximum to the second maximum is $\sim 1$ years during ascending phase of solar cycle 24 . Figure 4(b) shows the scatter graph between Halo CMEs and Dst Index during the ascending phases of solar cycles 23 and 24. It is observed that the Halo CMEs is the strongest correlation with Dst during the ascending phase of solar cycle 23 i.e. for the period 1996-2002. We have found correlation coefficient between Halo CMEs and Dst for solar cycle 23 is $\mathrm{R}=-0.85$ and for solar cycle 24 is $\mathrm{R}=-0.59$ during the ascending phases of solar cycles 23 and 24 . We have reported that the geomagnetic activity is depending on the CMEs during ascending phase of solar cycles 23 and 24. It is clear that the correlation coefficient between Halo CMEs and Dst for solar cycle 23 is greater than the solar cycle 24 .

From Figure 5 we have found that the annual average Dst is maximum for the ascending phase of solar cycle 23 in the period 2002 and for solar cycle 24 in the period 2012. We have reported that the maximum value of the annual average of 


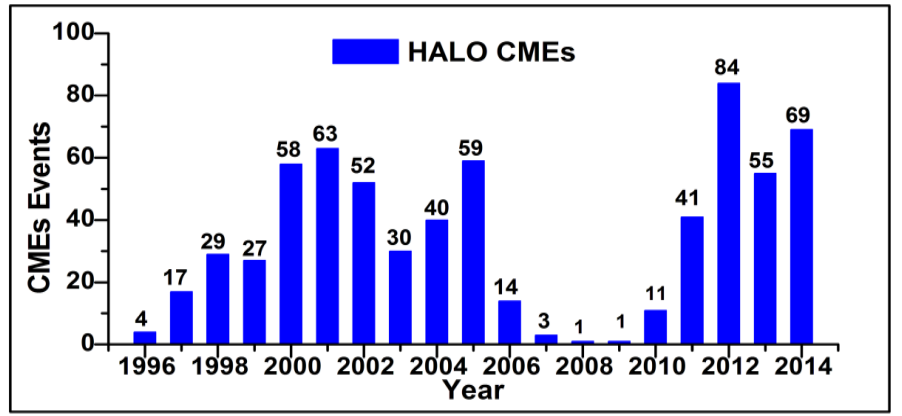

(a)
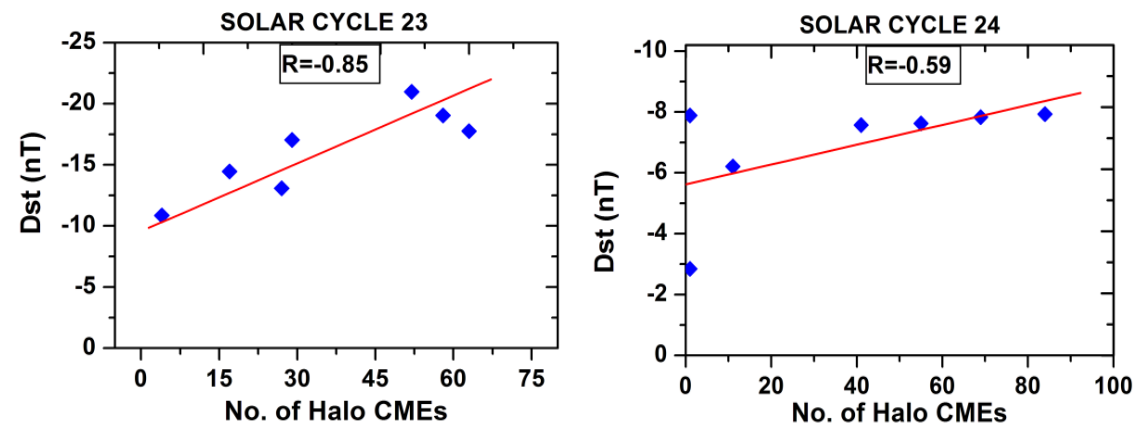

(b)

Figure 4. (a) Shows the annual variation of Halo CME during solar cycles 23 and 24 (b) Scatter graph between the annual value of Halo CMEs and Annual average value of Dst (nT) index for the ascending phase of solar cycles 23 and 24.

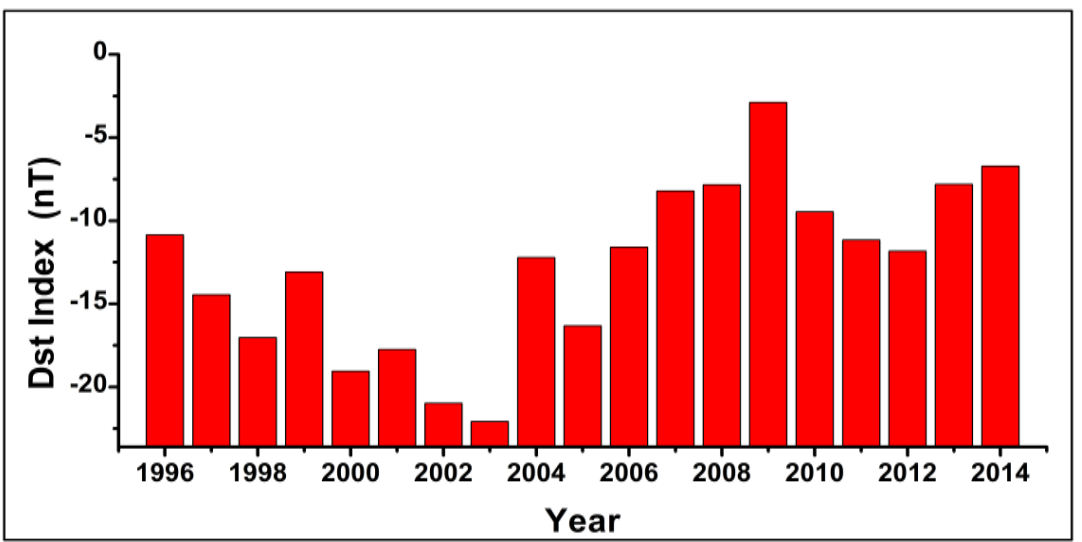

Figure 5. Shows the bar graph of the annual average of Dst Index for the year 1996-2014.

Dst is produced in the solar cycle 23 as compared to solar cycle 24 . We have observed that the annual average of Dst is maximum in the period 2009 during the solar cycle 24. It is observed that the Dst is depending on the CMEs during the study period [7].

Table 1 shows the fastest CMEs events ( $\geq 2200 \mathrm{~km} / \mathrm{sec})$ in ascending phase of solar cycle 23 and 24 . It is observed that CMEs with speed $\geq 2200 \mathrm{~km} / \mathrm{sec}$ are only 6 in solar cycle 23 as well as 4 in solar cycle 24 . In our discussions we adopt a simpler scheme: $D$ st $>-50 \mathrm{nT}$ denotes storm level (no storm); $-100 \mathrm{nT}<D$ st $\leq$ $-50 \mathrm{nT}$ denotes moderate storms while $D$ st $\leq-100 \mathrm{nT}$ denotes intense storms. 
Table 1. Shows the fastest CMEs events $(\geq 2200 \mathrm{~km} / \mathrm{sec})$ in ascending phase of solar cycle 23 and 24 .

\begin{tabular}{ccccc}
\hline First CME Appearance & $\begin{array}{c}\text { Time } \\
{[\mathrm{UT}]}\end{array}$ & $\begin{array}{c}\text { Speed } \\
{[\mathrm{km} / \mathrm{s}]}\end{array}$ & $\begin{array}{c}\text { Angular Width } \\
{[\mathrm{deg}]}\end{array}$ & $\begin{array}{c}\text { Geomagnetic } \\
\text { Storm }\end{array}$ \\
\hline $2000 / 05 / 12$ & $23: 26: 05$ & 2604 & Halo & No \\
$2000 / 11 / 25$ & $01: 31: 58$ & 2519 & Halo & No \\
$2001 / 04 / 02$ & $22: 06: 07$ & 2505 & Halo & No \\
$2001 / 04 / 10$ & $05: 30: 00$ & 2411 & Halo & Intense \\
$2001 / 04 / 18$ & $02: 30: 05$ & 2465 & Halo & Moderate \\
$2001 / 09 / 24$ & $10: 30: 59$ & 2402 & Halo & No \\
$2011 / 06 / 04$ & $22: 05: 02$ & 2425 & Halo & Moderate \\
$2012 / 01 / 27$ & $18: 27: 52$ & 2508 & Halo & No \\
$2012 / 03 / 07$ & $00: 24: 06$ & 2684 & Halo & Moderate \\
$2013 / 05 / 14$ & $01: 25: 51$ & 2625 & Halo & No \\
\hline
\end{tabular}

We analyze that these CMEs are producing geomagnetic storm or not. We have found 2 geomagnetic storms, (one moderate and one intense) during ascending phase of solar cycle 23 and 2 geomagnetic storms (both are moderate) during ascending phase of solar cycle 24 . We have also observed that there is no intense geomagnetic storm during ascending phase of solar cycle 24 but ascending phase of solar cycle 23 has one intense geomagnetic storm.

\section{Conclusions}

The main conclusions as follows: (1) we have found that the occurrence of fast CMEs $(>1000 \mathrm{~km} / \mathrm{sec})$ are only $1 \%$ in solar cycle 24 and $4 \%$ in solar cycle 23 . Therefore we have reported low solar activity in solar cycle 24 as compared to solar cycle 23. (2) Fast CMEs have a double peak structure in solar cycle 24 as well as solar cycle 23. (3) The occurrence of CMEs of higher width i.e. Halo CMEs is $3 \%$ in ascending phase of solar cycle 23 and $2 \%$ in ascending phase of solar cycle 24. (4) Double peak structure in the occurrence of Halo CME events is found in only solar cycles 24 . (5) The correlation between the annual occurrence of fast CMEs and an annual average of Dst index is lower in solar cycle 24 as compared to solar cycle 23. (6) The correlation coefficient between annual Halo CMEs and Dst index is lower in solar cycle 24 as compared to solar cycle 23. It is observed that the total Halo CMEs for ascending phase of solar cycle 23 is less than as compared to solar cycle 24 . We have suggested that the speed of CMEs with fast and Halo are responsible for producing strong geomagnetic storms. (6) On the basis of correlation, the geo-effectiveness of CMEs events i.e. Earth directed to CMEs for ascending phase of solar cycles 24 are less than in solar cycle 23, for our selected period (whereas the total number of CMEs in ascending phase of solar cycle 24 is greater than the ascending phase of solar cycle 23). We have reported that the Fast CMEs and Halo CMEs with Dst are strong 
correlation during the ascending phase of solar cycles 23 and 24 . Therefore we have concluded that in the above discussion the Dst (nT) have more negative value i.e. major geomagnetic storms are produced. We have reported that the fastest CMEs events $(\geq 2200 \mathrm{~km} / \mathrm{sec}$ ) are occurred Halo CMEs with an angular width $\left(360^{\circ}\right)$ for ascending phase of solar cycle 23 is larger as compared to cycle 24 . We have concluded that the fastest CMEs events $(\geq 2200 \mathrm{~km} / \mathrm{sec})$ produce intense and moderate geomagnetic storms during ascending phase of solar cycle 23 in the same month (for intense 10/04/2001 and for moderate 18/04/2001) but in solar cycle 24 we have found time lag ( $\sim 09$ months) between two moderate geomagnetic storms (for first moderate 04/06/2011 and for second 07/03/2012).

\section{Acknowledgements}

We sincerely thank an anonymous referee for providing constructive comments and suggestions toward enhancing the quality and presentation of this paper.

\section{References}

[1] Tsurutani, B.T., Gonzalez, W.D., Tang, F., Akasofu, S.I. and Smith, E.J. (1988) Solar Wind Southward $B_{z}$ Features Responsible for Major Magnetic Storms of 1978-1979. Journal of Geophysical Research, 93, 8519. https://doi.org/10.1029/JA093iA08p08519

[2] Gosling, J.T. (1993) The Solar Flare Myth. Journal of Geophysical Research, 98, 18937. https://doi.org/10.1029/93JA01896

[3] Jadeja, A.K., Iyer, K.N., Vats, O.M. and Manoharan, P.K. (2008) Geo-Effectiveness of CMEs. Journal of Astrophys \& Astronomy, 29, 287-291. https://doi.org/10.1007/s12036-008-0038-6

[4] Shrivastava, N. and Venkatakrishnan, P. (2002) Relationship between CME Speed and Geomagnetic Storm Intensity. Geophysical Research Letter, 29, 1287.

[5] Gopalswami, N. (2006) Coronal Mass Ejections of Solar Cycle 23. Journal of Astrophysics and Astronomy, 27, 243-254. https://doi.org/10.1007/BF02702527

[6] Ji, E.Y., Moon, Y.J. and Kim, K.H. (2010) Statistical Comparison of Interplanetary Conditions Causing Intense Geomagnetic Storms (Dst $\leq-100 \mathrm{nT}$ ). Journal of Geophysical Research, 115, A10232. https://doi.org/10.1029/2009JA015112

[7] Richardson, I.G. (2013) Geomagnetic Activity during the Rising Phase of Solar Cycle 24. Journal of Space Weather and Space Climate, 3, A08. https://doi.org/10.1051/swsc/2013031

[8] Gopalswamy, N. (2007) Coronal Mass Ejections and Space Weather. Climate and Weather of the Sun-Earth System (CAWSES), Kyoto Symposium, 77-120.

[9] Gopalswamy, N. (2004) In the Sun and the Heliosphere as an Integrated System Chap. 8. Kluwer Academic Publisher, Boston, Mass, 201.

[10] Yashiro, S., Gopalswamy, N., Michalek, G., St. Cyr, O.C., Plunkett, S.P., Rich, N.B. and Howard, R.A. (2004) A Catalog of White Light Coronal Mass Ejections Observed by the SOHO Spacecraft. Journal of Geophysical Research, 109, A07105. https://doi.org/10.1029/2003JA010282

[11] Singh, P.R., Ahmad, S., Pandey, A.C., Saxena, A.K., Tiwari, C.M. and Mishra, A.P. (2017) Cosmic Ray Associated with Coronal Index and Solar Flare Index during Solar Cycle 22-23. International Journal of Astronomy and Astrophysics, 7, 
162-173. https://doi.org/10.4236/ijaa.2017.73013

[12] Gopalswamy, N., et al. (2015) The Peculiar Behavior of Halo Coronal Mass Ejections in Solar Cycle 24. The Astrophysical Journal, 804, L23.

[13] Howard, R.A., et al. (1982) The Observation of a Coronal Transient Directed at Earth. The Astrophysical Journal Letters, 263, L101. https://doi.org/10.1086/183932

[14] Gopalswamy, N., Yashiro, S., Krucker, S., Stenborg, G. and Howard, R.A. (2004) Intensity Variation of Large Solar Energetic Particle Events Associated with Coronal Mass Ejections. Journal of Geophysical Research, 109, 12105. https://doi.org/10.1029/2004JA010602

[15] Gopalswamy, N., et al. (2015) Short-Term Variability of the Sun-Earth System: An Overview of Progress Made during the CAWSES-II Period. In: Zank, G., Ed., Proc. IAC, in the Press.

[16] Gopalswamy, N., et al. (2015) CMEs during the Two Activity Peaks in Cycle 24 and Their Space Weather Consequences.

\section{Scientific Research Publishing}

Submit or recommend next manuscript to SCIRP and we will provide best service for you:

Accepting pre-submission inquiries through Email, Facebook, LinkedIn, Twitter, etc. A wide selection of journals (inclusive of 9 subjects, more than 200 journals)

Providing 24-hour high-quality service

User-friendly online submission system

Fair and swift peer-review system

Efficient typesetting and proofreading procedure

Display of the result of downloads and visits, as well as the number of cited articles

Maximum dissemination of your research work

Submit your manuscript at: http://papersubmission.scirp.org/

Or contactijaa@scirp.org 